\title{
Medicina popular de Iquique, Tarapacá
}

\author{
Popular medicine of Iquique, Tarapacá
}

\author{
I.M. Madaleno ${ }^{1 *}$ y J. Delatorre-Herrera ${ }^{2}$
}

\section{RESUMEN}

Durante los meses de enero y febrero de 2012 se realizó una investigación en la ciudad de Iquique, en la Región de Tarapacá, por un equipo luso-chileno. El objeto del estudio fue la evaluación de la flora medicinal utilizada por los residentes de la ciudad costera, por opción o por no tener acceso a la medicina convencional. La pesquisa hace parte de un proceso de evaluación del peso de la flora nativa en el consumo de los latinoamericanos, iniciado por el Instituto Tropical de Lisboa a fines del siglo pasado. Se han compilado datos referentes a diez ciudades del Nuevo Mundo, siendo Iquique la localidad elegida para documentar los cultivos urbanos de plantas que curan, en ambiente desértico.

Palabras clave: saberes medicinales tradicionales, aymara, Tarapacá

\begin{abstract}
The city of Iquique, located in the northern Chilean Region of Tarapacá, has been investigated in January-February 2012, by a joint Portuguese-Chilean team. The main aim of the fieldwork was the evaluation of the medicinal flora used by local residents, as an option or for having no access to conventional medicine. This research is part of an ongoing project developed in the Portuguese Tropical Institute of Lisbon from the end of last century onwards, aimed at assessing the weight of the native American species used in herbal remedies, all over Latin America. So far a total of ten cities have been researched in the New World, and Iquique has been selected to document the urban cultivation of healing plant species in a desert environment.
\end{abstract}

Key words: traditional medicinal knowledge, aymara, Tarapacá

\section{Introducción}

El primer Servicio Nacional de Salud, que está asociado al estado del Bienestar, fue creado en el Reino Unido en 1948. Su modelo fue la organización de asistencia mutua establecida en el País de Gales, desde 1890, por las familias de mineros, la cual se reveló imprescindible para asegurar la buena salud de las comunidades galesas durante la gran depresión de los años 1930 (Featherstone et al., 2012). Las asociaciones mutualistas proseguirían en otros continentes, como fue el caso de las judías en Palestina, después Israel (1948), con la intención de proveer a los agricultores y obreros más carenciados cuidados de salud primaria, así como tratamiento más especializado en dispensarios y hospitales (Shvarts, 1995).

En Chile como consecuencia de la crisis económica de 1983, el gobierno de la época privatizó parte de los actos médicos, creando sistemas privados de salud para la población de mayores recursos y manteniendo un sistema público para los más desposeídos; sin embargo, este último sistema no satisface las necesidades de los mismos (Gómez y Calderón, 2011). A pesar de ello, los indicadores del estado de la salud de la población en Chile se ubican entre los mejores del continente y muy cerca de los de países desarrollados (CEPAL, 2003).

Chile presenta una larga tradición de políticas sociales que han contribuido notoriamente a expandir la cobertura de la atención de salud y a elevar los indicadores sanitarios a los niveles que hoy presenta el país (Olavarría, 2004). Aunque en el nivel agregado los indicadores sobre el estado de salud de la población son relativamente favorables para Chile, ha habido intensas críticas al sistema público de salud al que acuden las personas de menores ingresos, en términos de la calidad y oportunidad de los servicios entregados. Dado que mantenerse sano es uno de los activos con que cuentan los

1 Instituto de Investigaciones Científicas Tropicales, Lisboa, Portugal. Email: isabelmadaleno8@gmail.com

2 Facultad de Recursos Naturales Renovables. Universidad Arturo Prat, Iquique, Chile.

* Autor para correspondencia.

Fecha de Recepción: 22 Enero, 2013.

Fecha de Aceptación: 25 Febrero, 2013. 
pobres (para poder trabajar), los problemas que ellos tengan en acceder a atención de salud cuando la necesitan podrían traducirse en obstáculos serios a sus esfuerzos por superar el estado de pobreza (Olavarría, 2005).

Los pueblos originarios de Chile han utilizado diversas plantas para tratar problemas de salud. Este conocimiento fue transmitido a los campesinos, quienes heredaron esta tradición que se extiende hasta la actualidad a nivel de la población. Con el objetivo de aprovechar al máximo esta sabiduría, el Ministerio de Salud (Minsal) ha integrado 103 plantas medicinales chilenas a la Política Nacional de Medicamentos. De esta forma, el sistema de salud chileno pasa a incorporar la medicina natural como un complemento de los tratamientos occidentales tradicionales. Esta iniciativa va de la mano con las estrategias planteadas por la Organización Mundial de la Salud (OMS), que recomienda integrar los conocimientos originales y populares a la salud pública, además de regularlos y facilitar su acceso para la población (Fucoa, 2010).

Iquique se localiza en la región de Tarapacá con una gran influencia de la etnia aymara, la cual tiene una gran tradición en el uso de hierbas medicinales nativas. Al respecto, Acevedo y Delatorre (2012) han publicado un listado de plantas medicinales altoandinas de la I Región de Tarapacá, en el cual se describen 64 plantas nativas que son usadas en la medicina tradicional de la zona. El artículo que se presenta enfoca todas las especies consumidas por los iquiqueños, independientemente de su origen geográfico. Se divide en tres partes: en la primera se ubica el área de estudio, se enumeran los objetivos de la pesquisa, así como los materiales y métodos usados; en la segunda se discuten los resultados obtenidos; finalmente se encierra con las conclusiones seguidas del listado de todas las especies de uso terapéutico, las partes consumidas y su manejo por los iquiqueños.

\section{El área de estudio}

Iquique es una ciudad con 284.539 habitantes (INE, 2012) cuya principal base económica se relaciona con la minería, pero es también un puerto franco con un importante contingente de residentes ocupados en actividades del sector terciario (Escolano et al., 2007). También existe un porcentaje importante de inmigrantes de países como India, China y Pakistán, que están bien integrados y contribuyen a la economía local y nacional. Esta ciudad se emplaza en el borde costero del desierto de Atacama, por lo que su clima es desértico-costero nuboso. Iquique tiene $18^{\circ}$ de temperatura media anual, una precipitación total de $1,9 \mathrm{~mm}$ y presenta nubosidad matinal debido a la corriente fría de Humboldt, fenómeno denominado camanchaca. La ciudad se ubica en la Primera Región de Tarapacá, entre un acantilado amurallado (el relieve costero) y el mar (Sánchez y Morales, 2004).

El presente estudio forma parte de una amplia investigación destinada a evaluar en el mundo el uso de las plantas medicinales a nivel urbano. El cuadro $\mathrm{N}^{\circ} 1$ presenta las urbes investigadas desde hace 15 años por el Instituto Tropical de Lisboa, en América Latina. En las muestras de Santiago de Chile, el Instituto Tropical de Lisboa trabajó juntamente con la Universidad de Chile y, en Iquique, con la Universidad Arturo Prat. En Lima, el trabajo tuvo el apoyo de la Pontificia Universidad Católica del Perú. En Cuernavaca y Puebla, la Universidad Nacional Autónoma de México. En Argentina, el convenio se estableció con la Universidad Nacional de Río Cuarto. En Brasil, el Instituto Tropical contó con el apoyo del Núcleo de Altos Estudios Amazónicos, en Belém. En Uruguay, Cuba y Costa Rica no se establecieron acuerdos formales con universidades nacionales.

\section{Objetivos}

Los objetivos específicos de la investigación son los que siguen:

1) Evaluar la importancia de la herbolaria nativa, según las preferencias de consumo de las poblaciones de la ciudad de Iquique;

2) Evaluar el peso de la influencia europea colonial en los usos y especies medicinales introducidas;

3) Compilar el recetario de hierbas y plantas medicinales usadas en cuatro enfermedades específicas: diabetes, artritis, enfermedades de los ojos y cáncer (Madaleno, 2007).

\section{Materiales y Métodos}

Como metodología se utilizó la misma usada en la recolección de información primaria en ocho países y diez aglomeraciones urbanas de América Latina y constó de trabajo de campo realizado por la primera autora, por medio de entrevistas a tres grupos de informantes (Madaleno, 2011ab, 2012). Esta consistió en: 
Cuadro $\mathrm{N}^{\circ}$ 1. Información primaria reunida en América Latina.

\begin{tabular}{|c|c|c|c|c|c|}
\hline Urbes y metrópolis & Rango & $\begin{array}{l}\text { Localización } \\
\text { geográfica }\end{array}$ & $\begin{array}{l}\text { Muestras } \\
\left(\mathrm{N}^{\circ} \text { entrev.) }\right.\end{array}$ & Años & $\begin{array}{c}\mathrm{N}^{\mathrm{o}} \text { de especies } \\
\text { medicinales }\end{array}$ \\
\hline $\begin{array}{l}\text { Región metropolitana } \\
\text { central de México }\end{array}$ & $\begin{array}{c}\text { Capital nacional y dos } \\
\text { estaduales }\end{array}$ & $\begin{array}{l}\text { Altiplano mexicano y } \\
\text { valles interiores }\end{array}$ & 155 & 2004, 2006 & 70 \\
\hline $\begin{array}{c}\text { Región metropolitana } \\
\text { de Lima }\end{array}$ & Capital nacional & $\begin{array}{l}\text { Desierto costero } \\
\text { peruano }\end{array}$ & 34 & 2006 & 60 \\
\hline $\begin{array}{c}\text { Región Metropolitana } \\
\text { de Santiago }\end{array}$ & Capital nacional & $\begin{array}{l}\text { Depresión interior } \\
\text { chilena temperada }\end{array}$ & 132 & $\begin{array}{l}2002,2003, \\
2005\end{array}$ & 70 \\
\hline $\begin{array}{l}\text { Región metropolitana de } \\
\text { La Habana }\end{array}$ & Capital nacional & $\begin{array}{l}\text { Llanura costera } \\
\text { tropical caribeña }\end{array}$ & 50 & 2009 & 60 \\
\hline $\begin{array}{c}\text { Región metropolitana de } \\
\text { San José }\end{array}$ & Capital nacional & $\begin{array}{l}\text { Valle interior } \\
\text { tropical costarricense }\end{array}$ & 43 & 2009 & 60 \\
\hline $\begin{array}{c}\text { Región metropolitana de } \\
\text { Belem }\end{array}$ & Capital estadual & Amazonia brasileña & 570 & 1998,2005 & 140 \\
\hline Ciudad de S. Luis & Capital estadual & $\begin{array}{c}\text { Isla fluvial } \\
\text { costera tropical }\end{array}$ & 100 & 2010 & 109 \\
\hline Río Cuarto & Ciudad mediana & $\begin{array}{l}\text { Pampa argentina } \\
\text { temperada }\end{array}$ & 100 & 2011 & 125 \\
\hline Colonia del Sacramento & Ciudad pequeña & Llanura fluvial temperada & 50 & 2011 & 70 \\
\hline Iquique & Ciudad mediana & Desierto costero chileno & 75 & 2012 & 85 \\
\hline
\end{tabular}

Fuente: Madaleno 2007, 2011a, 2011b y Madaleno y Montero 2011.

1) Establecer los ciudadanos que cultivan plantas con usos terapéuticos en su jardín;

2) Cuantificar los comerciantes que venden tés y preparados a los que tienen fe en la terapia con plantas;

3) Determinar los acopiadores de hierbas y curanderos, así como médicos naturistas que utilizan hierbas naturales.

Fueron 48 los entrevistados en el primer grupo de informantes, por medio de entrevistas semiestructuradas, por cuanto se utiliza un mismo cuestionario para todas las muestras, en un proceso hecho al azar, de calle en calle, puerta a puerta, en seis barrios, los cuales fueron discriminados según las siguientes categorías:
A) Clase media-alta o de alto presupuesto: 1) Barrio Cavancha (2 entrevistas);

B) 2) Condominio Huayquique (cinco entrevistas).

C) Clase media y media-baja:

3) Conjunto Gabriela Mistral (6 entrevistas);

4) Conjunto Reynamar (16 entrevistas);

5) Conjunto Rey del Mar (10 entrevistas);

6) Conjunto Magisterio (9 entrevistas).

La muestra no contempla los pobres, ni en general las clases sociales de bajo presupuesto, ya que residen en departamentos o no poseen condiciones para cultivar especies de uso terapéutico, dentro de la ciudad. 
El segundo grupo de informantes totalizó 22 comerciantes, entrevistados en tiendas de barrio, supermercados con venta de hierbas, herboristerías, en el Terminal Agropecuario, en la Feria Sur, en el Mercado Central. Finalmente, en el último grupo se entrevistaron, en el estudio vertiente, un acopiador de hierbas de Pica y una artesana de productos naturales. A estos dos informantes se agregaron tres acopiadoras de la precordillera y valles altos (Sibaya), del altiplano (Colchane y Cariquima), que abastecen plantas de la medicina tradicional aymara a los mercaderes y las recomiendan a quienes no tienen acceso a la medicina convencional.

El proceso de investigación incluye, con posterioridad, la identificación de las especies en las universidades e instituciones participantes, donde se deposita el material recolectado.

\section{Presentación y Discusión de los Resultados}

\section{Plantas medicinales y sus usos en Iquique}

El cuadro 2 discrimina los entrevistados por nivel etario, reunidos en la muestra realizada en Tarapacá. Como primer resultado reseñable se prueba que están en aumento los iquiqueños que se dedican a cultivar plantas medicinales en sus jardines y patios traseros, que corresponden a $60,4 \%$ de los entrevistados que declararon cultivar ese tipo de especies terapéuticas hace menos de diez años. En conformidad, el 36,4\% de las herboristerías y de los mercaderes vendían flora medicinal hace menos de una década, en este caso, sobre todo a poblaciones de baja renta, hecho reseñable en la observación de clientes, durante las entrevistas a vendedores del mercado central.

Esto nos conduce al segundo resultado que estriba del hecho que la confianza en la cura de enfermedades utilizando plantas está en progresión. Tal observación está conforme con los demás estudios realizados en América Latina, hasta el presente, donde la sucesión de crisis financieras (Dadush y Shaw, 2011, Santandreu et al., 2009) promueve la búsqueda de alternativas a la medicina convencional, más cara, cada día menos universal, sobre todo en caso de molestias menos graves y de malestares súbitos (Madaleno 2011b, Madaleno y Montero, 2011, Who, 2009).

El tercer resultado prueba, con naturalidad, esta conclusión respecto de los iquiqueños, pues el $43 \%$ de las hierbas cultivadas, vendidas o recomendadas, son digestivas, infusiones que se toman contra gastritis, úlceras y afecciones gástricas diversas. Cerca del $21 \%$ de las plantas consumidas en Iquique son utilizadas contra resfríos y tos. El 17\% son analgésicos y el $11 \%$ son hemostáticos y cicatrizantes naturales.

Cuadro 2. Información primaria reunida en Tarapacá.

\begin{tabular}{|c|c|c|c|c|}
\hline \multirow{2}{*}{$\begin{array}{c}\text { Clases } \\
\text { años }\end{array}$} & \multicolumn{2}{|c|}{$\begin{array}{l}\text { Edades de los entrevistados } \\
\qquad\left(\mathrm{N}^{\circ}\right)\end{array}$} & \multicolumn{2}{|c|}{$\begin{array}{l}\text { Tiempo de cultivo o de actividad } \\
\qquad\left(\mathrm{N}^{\circ}\right)\end{array}$} \\
\hline & $\mathrm{H}$ & M & $\mathrm{H}$ & M \\
\hline$<5$ & - & - & 2 & 14 \\
\hline $5-10$ & - & - & 4 & 18 \\
\hline $10-14$ & - & - & 3 & 13 \\
\hline $15-19$ & - & - & 1 & 2 \\
\hline $20-24$ & 1 & 2 & 1 & 2 \\
\hline $25-29$ & 1 & 3 & 0 & 1 \\
\hline $30-34$ & 2 & 7 & 3 & 3 \\
\hline $35-39$ & 0 & 5 & 0 & 3 \\
\hline $40-44$ & 1 & 6 & 1 & 2 \\
\hline $45-49$ & 1 & 5 & 0 & 0 \\
\hline $50-54$ & 3 & 8 & 0 & 0 \\
\hline $55-59$ & 2 & 5 & 0 & 0 \\
\hline $60-64$ & 2 & 9 & 1 & 0 \\
\hline $65-69$ & 1 & 6 & 0 & 1 \\
\hline $70-74$ & 1 & 2 & - & - \\
\hline $75-79$ & 0 & 1 & - & - \\
\hline $80-84$ & 0 & 0 & - & - \\
\hline $85-89$ & 1 & 0 & - & - \\
\hline Total & 16 & 59 & 16 & 59 \\
\hline
\end{tabular}

Fuente: Muestra de 2012. 
El Cuadro 3 presenta, con gran detalle, todas las especies recolectadas en la muestra y sus aplicaciones terapéuticas. En ella se discrimina el nombre vernáculo y la identificación botánica, usando la norma de los Missouri Botanical Gardens (MBG, 2012). Como cuarto resultado subrayamos el registro de treinta y nueve familias distintas, con predominancia de especies herbáceas, como
Asteraceae (11 plantas) y Lamiaceae (8). Señalamos aun la importancia de Apiaceae, con siete nombres vernáculos, que corresponden a las preferencias en los consumos de especies terapéuticas por los iquiqueños. Fueron ochenta y cinco especies registradas en la muestra, de las cuales tan sólo dieciocho (18) se han encontrado y fotografiado en los jardines y patios traseros.

Cuadro 3. Lista de plantas medicinales de Iquique, Chile

\begin{tabular}{|c|c|c|c|c|}
\hline $\begin{array}{l}\text { Nombre } \\
\text { vernáculo }\end{array}$ & Frecuencia & $\begin{array}{l}\text { Nombre científico } \\
\text { FAMILIA }\end{array}$ & Parte usada & Aplicaciones terapéuticas \\
\hline Ajenjo & 2 & $\begin{array}{l}\text { Artemisia absinthium L. } \\
\text { ASTERACEAE }\end{array}$ & Hojas & $\begin{array}{c}\text { Gota, gastritis, diurético, reumatismo, } \\
\text { diabetes. }\end{array}$ \\
\hline Ajo & 2 & $\begin{array}{l}\text { Allium sativum } \mathrm{L} \text {. } \\
\text { AMARYLLIDACEAE }\end{array}$ & Bulbo & Descongestiona las vías respiratorias. \\
\hline Alfalfa & 1 & $\begin{array}{l}\text { Medicago sativa } \mathrm{L} \text {. } \\
\text { FABACEAE }\end{array}$ & Hojas & $\begin{array}{l}\text { Enfermos del corazón, mejora } \\
\text { circulación sanguínea. }\end{array}$ \\
\hline Aloe & 32 & $\begin{array}{l}\text { Aloe vera }(\mathrm{L} .) \text { Burm. } \mathrm{f} . \\
\text { Aloe barbadensis Mill. } \\
\text { AMARANTHACEAE }\end{array}$ & $\begin{array}{l}\text { Visco } \\
\text { y cáscara }\end{array}$ & $\begin{array}{l}\text { Cicatrizante, heridas, machucaduras, } \\
\text { hongos de uñas, pie de atleta, acné, } \\
\text { antiinflamatorio, psoriasis, quemaduras, } \\
\text { reumatismo, caída del pelo, antiséptico, } \\
\text { mejora el cutis (ext.), gastritis, } \\
\text { digestivo, contra estreñimiento, úlceras } \\
\text { estomacales, laxante (int.) }\end{array}$ \\
\hline Amaranto & 2 & $\begin{array}{l}\text { Amaranthus viridis } \mathrm{L} \text {. } \\
\text { AMARANTHACEAE }\end{array}$ & Semillas & Energizante, baja colesterol \\
\hline Anís & 2 & $\begin{array}{l}\text { Pimpinella anisum } \mathrm{L} \text {. } \\
\text { APIACEAE }\end{array}$ & Parte aérea & $\begin{array}{l}\text { Calmante, carminativo, estomacal, } \\
\text { digestivo, aumenta leche materna, } \\
\text { contra cólicos de guaguas, bronquitis. }\end{array}$ \\
\hline Apio & 3 & $\begin{array}{l}\text { Apium graveolens } \mathrm{L} . \\
\text { APIACEAE }\end{array}$ & Bulbo & Contra cólicos de guaguas. \\
\hline Bailahuén & 3 & $\begin{array}{c}\text { Haplopappus baylahuen Remy } \\
\text { ASTERACEAE }\end{array}$ & Parte aérea & $\begin{array}{l}\text { Dolores de estómago, digestivo, } \\
\text { carminativo, gastritis. }\end{array}$ \\
\hline Berro & 1 & $\begin{array}{c}\text { Rorippa nasturtium-aquaticum (L.) } \\
\text { Hayek } \\
\text { BRASSICACEAE }\end{array}$ & Hojas & Carminativo. \\
\hline Boldo & 2 & $\begin{array}{l}\text { Peumus boldus Molina } \\
\text { MONIMIACEAE }\end{array}$ & Hojas & $\begin{array}{l}\text { Problemas de hígado, vesícula biliar, } \\
\text { digestivo, estomacal, diurético, jaqueca. }\end{array}$ \\
\hline Borraja & 3 & $\begin{array}{l}\text { Borago officinalis L. } \\
\text { BORAGINACEAE }\end{array}$ & Parte aérea & $\begin{array}{l}\text { Carminativo, estomacal, resfríos, fiebre } \\
\text { (int.), retraso de las reglas, herpes (ext.). }\end{array}$ \\
\hline Caléndula & 1 & $\begin{array}{l}\text { Calendula officinalis L. } \\
\text { ASTERACEAE }\end{array}$ & Flores & $\begin{array}{l}\text { Antisépticas, antiinflamatorias, } \\
\text { cicatrizante, antiacné. }\end{array}$ \\
\hline Canela & 2 & $\begin{array}{c}\text { Cinnamomum zeylanicum Blume } \\
\text { LAURACEAE }\end{array}$ & Cáscara & Carminativa. \\
\hline Cebolla & 1 & $\begin{array}{c}\text { Allium cepa } \mathrm{L} \text {. } \\
\text { AMARYLLIDACEAE }\end{array}$ & Bulbo & Expectorante, antiasmático. \\
\hline $\begin{array}{l}\text { Chachacoma } \\
\text { negra }\end{array}$ & 4 & $\begin{array}{l}\text { Senecio eriophyton Remy } \\
\text { ASTERACEAE }\end{array}$ & Parte aérea & $\begin{array}{l}\text { Regula presión arterial, alivio dolores } \\
\text { estomacales, mal de altura, mejora } \\
\text { memoria. }\end{array}$ \\
\hline
\end{tabular}




\begin{tabular}{|c|c|c|c|c|}
\hline $\begin{array}{l}\text { Nombre } \\
\text { vernáculo }\end{array}$ & Frecuencia & $\begin{array}{l}\text { Nombre científico } \\
\text { FAMILIA }\end{array}$ & Parte usada & Aplicaciones terapéuticas \\
\hline Cedrón & 17 & $\begin{array}{l}\text { Aloysia triphylla Royle } \\
\text { VERBENACEAE }\end{array}$ & Hojas & Estómago, digestivo, estrés. \\
\hline Cilantro & 1 & $\begin{array}{l}\text { Coriandrum sativum } \mathrm{L} \text {. } \\
\text { APIACEAE }\end{array}$ & Hojas & Digestivo. \\
\hline Ciprés & 1 & $\begin{array}{l}\text { Cupressus lusitanica Mill. } \\
\text { CUPRESSACEAE }\end{array}$ & Hojas & Problemas de próstata. \\
\hline Coca & 10 & $\begin{array}{l}\text { Erythroxylum coca Lam. } \\
\text { ERYTHROXYLACEAE }\end{array}$ & Hojas & $\begin{array}{l}\text { Dolor de estómago, dolor de cabeza, } \\
\text { mal de altura, regula presión arterial, } \\
\text { contra estrés. }\end{array}$ \\
\hline Cola de caballo & 7 & $\begin{array}{l}\text { Equisetum giganteum } \mathrm{L} . \\
\text { E. arvense } \mathrm{L} . \\
\text { EQUISETACEAE }\end{array}$ & Palos & $\begin{array}{l}\text { Diurético, gota, riñones, } \\
\text { antihemorrágico. }\end{array}$ \\
\hline Culén & 1 & $\begin{array}{c}\text { Otholobium glandulosum (L.) J.W. } \\
\text { Grimes } \\
\text { FABACEAE }\end{array}$ & Hojas & $\begin{array}{l}\text { Colon irritable, diarrea crónica, } \\
\text { diabetes. }\end{array}$ \\
\hline Eneldo & 1 & $\begin{array}{l}\text { Anethum graveolens } \mathrm{L} \text {. } \\
\text { APIACEAE }\end{array}$ & Parte aérea & Desinflama, mejora tránsito intestinal. \\
\hline Estevia & 1 & $\begin{array}{c}\text { Stevia boliviensis Sch. Bip. ex Griseb. } \\
\text { ASTERACEAE }\end{array}$ & Hojas & Diabetes, endulzante natural \\
\hline Eucalipto & 5 & $\begin{array}{l}\text { Eucalyptus globulus Labill. } \\
\text { MYRTACEAE }\end{array}$ & $\begin{array}{l}\text { Hojas } \\
\text { y semillas }\end{array}$ & $\begin{array}{c}\text { Antigripal, febrífugo, expectorante, tos, } \\
\text { dolor de garganta (hoja), antiséptico } \\
\text { (semilla). }\end{array}$ \\
\hline Flor de Saúco & 1 & $\begin{array}{l}\text { Sambucus nigra L. } \\
\text { ADOXACEAE }\end{array}$ & Flor & Pectoral, resfríos, ciática, neuralgia. \\
\hline Flor de Yareta & 15 & $\begin{array}{c}\text { Azorella compacta } \text { Phil. } \\
\text { APIACEAE }\end{array}$ & Flor & Diabetes, catarro pulmonar. \\
\hline Ginkgo biloba & 1 & $\begin{array}{l}\text { Ginkgo biloba } \mathrm{L} . \\
\text { GINKGOACEAE }\end{array}$ & Hojas & Memoria, rejuvenece. \\
\hline Hibiscos & 1 & $\begin{array}{l}\text { Hibiscus sabdariffa } \mathrm{L} . \\
\text { MALVACEAE }\end{array}$ & Flor & Digestivo. \\
\hline Hierbaluisa & 18 & $\begin{array}{c}\text { Cymbopogon citratus (DC.) Stapf } \\
\text { POACEAE }\end{array}$ & Hojas & Digestivo, contra resfríos. \\
\hline Hinojo & 4 & $\begin{array}{l}\text { Foeniculum vulgare Mill. } \\
\text { APIACEAE }\end{array}$ & $\begin{array}{l}\text { Semillas } \\
\text { y hojas }\end{array}$ & $\begin{array}{c}\text { Digestivo, carminativo, expectorante, } \\
\text { despeja las vías respiratorias, cáncer a } \\
\text { la próstata. }\end{array}$ \\
\hline Jengibre & 1 & $\begin{array}{l}\text { Zingiber officinale } \text { Roscoe } \\
\text { ZINGIBERACEAE }\end{array}$ & Bulbo & Tos. \\
\hline Kipa hembra & 1 & $\begin{array}{l}\text { Fabiana denudata } \text { Miers } \\
\text { SOLANACEAE }\end{array}$ & Parte aérea & Tos. \\
\hline Lampaya & 9 & $\begin{array}{l}\text { Lampayo medicinalis F. Phil. } \\
\text { VERBENACEAE }\end{array}$ & Hojas & $\begin{array}{l}\text { Riñones, diurético, cistitis, cálculos } \\
\text { renales, problemas de próstata, dolor de } \\
\text { huesos, resfríos. }\end{array}$ \\
\hline Laurel & 1 & $\begin{array}{l}\text { Laurus nobilis L. } \\
\text { LAURACEAE }\end{array}$ & Hojas & $\begin{array}{l}\text { Digestivo, carminativo, relajante, } \\
\text { bronquios. }\end{array}$ \\
\hline Lengua de gallina & 1 & $\begin{array}{c}\text { Chuquiraga atacamensis Kuntze } \\
\text { ASTERACEAE }\end{array}$ & Parte aérea & $\begin{array}{l}\text { Cicatrizante, heridas, problemas de } \\
\text { huesos, fracturas }\end{array}$ \\
\hline Limón & 2 & $\begin{array}{l}\text { Citrus medica } \mathrm{L} . \\
\text { RUTACEAE }\end{array}$ & Fruto & Resfríos. \\
\hline Llantén & 14 & $\begin{array}{l}\text { Plantago major } \mathrm{L} . \\
\text { PLANTAGINACEAE }\end{array}$ & Hojas & $\begin{array}{l}\text { Estómago, carminativo, hepático (int.), } \\
\text { cicatrizante (ext.). }\end{array}$ \\
\hline
\end{tabular}




\begin{tabular}{|c|c|c|c|c|}
\hline $\begin{array}{l}\text { Nombre } \\
\text { vernáculo }\end{array}$ & Frecuencia & $\begin{array}{l}\text { Nombre científico } \\
\text { FAMILIA }\end{array}$ & Parte usada & Aplicaciones terapéuticas \\
\hline Maca & 2 & $\begin{array}{c}\text { Lepidium peruvianum G. Chacón } \\
\text { BRASSICACEAE }\end{array}$ & Bulbo & Osteoporosis. \\
\hline Malva rosa & 2 & $\begin{array}{c}\text { Pelargonium odoratissimum (L.) } \\
\text { L'Hér. } \\
\text { GERANIACEAE }\end{array}$ & Parte aérea & Estómago, mareo. \\
\hline Manzanilla & 16 & $\begin{array}{l}\text { Matricaria chamomilla L. } \\
\text { ASTERACEAE }\end{array}$ & Parte aérea & $\begin{array}{l}\text { Indigestión, dolor de cabeza, de panza, } \\
\text { facilita la menstruación, relajante (int.), } \\
\text { antirreumático, cicatrizante (ext.). }\end{array}$ \\
\hline Maqui & 1 & $\begin{array}{l}\text { Aristotelia chilensis (Molina) Stuntz } \\
\text { ELAEOCARPACEAE }\end{array}$ & Hojas & Antioxidante. \\
\hline Mate & 2 & $\begin{array}{l}\text { Ilex paraguariensis A. St.-Hil. } \\
\text { AQUIFOLIACEAE }\end{array}$ & Hojas & Adelgazante. \\
\hline Matico & 12 & $\begin{array}{l}\text { Buddleja globosa Hope } \\
\text { SCROPHULARIACEAE }\end{array}$ & Hojas & $\begin{array}{c}\text { Colon irritable, regulador de colesterol, } \\
\text { digestivo, gastritis, diabetes, úlceras } \\
\text { estomacales, antiinflamatorio (int.), } \\
\text { hemostático, cicatrizante (ext.). }\end{array}$ \\
\hline Melisa & 4 & $\begin{array}{l}\text { Melissa officinalis L. } \\
\text { LAMIACEAE }\end{array}$ & Hojas & $\begin{array}{l}\text { Insomnio, antiestrés, analgésico, } \\
\text { palpitaciones, dolor de cabeza. }\end{array}$ \\
\hline Menta & 23 & $\begin{array}{l}\text { Mentha x piperita } \mathrm{L} . \\
\text { Mentha viridis }(\mathrm{L} .) \mathrm{L} . \\
\text { LAMIACEAE }\end{array}$ & Hojas & $\begin{array}{l}\text { Carminativa, digestiva, resfrío, dolor } \\
\text { de cabeza, de estómago, de panza, } \\
\text { relajante, antioxidante }\end{array}$ \\
\hline Molle & 2 & $\begin{array}{l}\text { Schinus molle L. } \\
\text { ANACARDIACEAE }\end{array}$ & Hojas & $\begin{array}{c}\text { Analgésico, cólico (hojas), cicatrizante } \\
\text { y regenerador de tejidos cutáneos (ceiba } \\
\text { o lágrima de Molle). }\end{array}$ \\
\hline Morera & 1 & $\begin{array}{l}\text { Morus tinctoria } \mathrm{L} . \\
\text { MORACEAE }\end{array}$ & Hojas & Diabetes \\
\hline Muña & 2 & $\begin{array}{c}\text { Minthostachys setosa (Briq.) Epling } \\
\text { LAMIACEAE }\end{array}$ & Hojas & Antiséptico (ext.), colon irritable (int.). \\
\hline $\begin{array}{l}\text { Ñacatula o } \\
\text { Ñakat'ula }\end{array}$ & 1 & $\begin{array}{l}\text { Baccharis santelices Phil. } \\
\text { ASTERACEAE }\end{array}$ & Parte aérea & Cólicos. \\
\hline Naranjo & 2 & $\begin{array}{l}\text { Citrus aurantium } \mathrm{L} . \\
\text { RUTACEAE }\end{array}$ & $\begin{array}{c}\text { Hojas y cáscaras } \\
\text { de fruto }\end{array}$ & $\begin{array}{l}\text { Somnífero, resfríos, calmante (hojas), } \\
\text { celulitis (cáscaras). }\end{array}$ \\
\hline Orégano & 14 & $\begin{array}{l}\text { Origanum vulgare L. } \\
\text { LAMIACEAE }\end{array}$ & Hojas & $\begin{array}{l}\text { Carminativa, hinchazón de guatita, } \\
\text { relajante (int.), verrugas, antiviral (ext.). }\end{array}$ \\
\hline Paico & 10 & $\begin{array}{l}\text { Chenopodium ambrosioides } \mathrm{L} . \\
\text { AMARANTHACEAE }\end{array}$ & $\begin{array}{l}\text { Semillas } \\
\text { y hojas }\end{array}$ & $\begin{array}{l}\text { Digestiva, parásitos, empacho de } \\
\text { guaguas, antidiarreico, dolor de } \\
\text { estómago. }\end{array}$ \\
\hline Palta o Palto & 2 & $\begin{array}{l}\text { Persea americana Mill. } \\
\text { LAURACEAE }\end{array}$ & Fruto y hojas & $\begin{array}{l}\text { Digestivo, caída y brillo del cabello } \\
\text { (fruto), tos (hojas). }\end{array}$ \\
\hline Pasiflora & 2 & $\begin{array}{l}\text { Passiflora incarnata } \mathrm{L} \text {. } \\
\text { PASSIFLORACEAE }\end{array}$ & Flores & $\begin{array}{l}\text { Sedante, hipnótica, antiespasmódica, } \\
\text { antiestrés. }\end{array}$ \\
\hline Pata de vaca & 1 & $\begin{array}{l}\text { Bauhinia forficata Link } \\
\text { FABACEAE }\end{array}$ & Hojas & Diabetes. \\
\hline Pepino & 1 & $\begin{array}{l}\text { Cucumis sativus } \mathrm{L} . \\
\text { CUCURBITACEAE }\end{array}$ & Fruto & Mejora el cutis. \\
\hline Perejil & 4 & $\begin{array}{c}\text { Petroselinum crispum (Mill.) Fuss } \\
\text { APIACEAE }\end{array}$ & Toda la planta & Antioxidante, adelgazante. \\
\hline Pichi & 1 & $\begin{array}{l}\text { Fabiana imbricata Ruiz \& Pav. } \\
\text { SOLANACEAE }\end{array}$ & Parte aérea & $\begin{array}{c}\text { Diurético, sedante, riñones, problemas a } \\
\text { la próstata (int.), cicatrizante, antiséptico } \\
\text { (ext.). }\end{array}$ \\
\hline
\end{tabular}




\begin{tabular}{|c|c|c|c|c|}
\hline $\begin{array}{l}\text { Nombre } \\
\text { vernáculo }\end{array}$ & Frecuencia & $\begin{array}{l}\text { Nombre científico } \\
\text { FAMILIA }\end{array}$ & Parte usada & Aplicaciones terapéuticas \\
\hline Pingo-pingo & 2 & $\begin{array}{l}\text { Ephedra andina Poepp. \& Endl. } \\
\text { EPHEDRACEAE }\end{array}$ & Cáscaras & Problemas a la próstata. \\
\hline Poleo & 3 & $\begin{array}{l}\text { Mentha pulegium L. } \\
\text { LAMIACEAE }\end{array}$ & Hojas & $\begin{array}{c}\text { Dolores de estómago, de cabeza, tos, } \\
\text { nervios, laxante. }\end{array}$ \\
\hline Pupusa & 4 & $\begin{array}{l}\text { Arenaria serpens Kunth } \\
\text { CARIOPHYLLACEAE }\end{array}$ & Parte aérea & $\begin{array}{l}\text { Estómago inflamado, hígado, colon } \\
\text { irritable, digestivo, regula presión } \\
\text { arterial, hepática. }\end{array}$ \\
\hline Queñoa & 6 & $\begin{array}{c}\text { Polylepis tarapacana Phil. } \\
\text { ROSACEAE }\end{array}$ & Cáscaras & Tos, asma, bronquitis, resfríos. \\
\hline Quinua & 5 & $\begin{array}{l}\text { Chenopodium quinoa Willd. } \\
\text { AMARANTHACEAE }\end{array}$ & Semillas & Baja colesterol. \\
\hline Rica-rica & 3 & $\begin{array}{c}\text { Alcantholippia deserticola Mill. } \\
\text { ROSACEAE }\end{array}$ & Parte aérea & $\begin{array}{l}\text { Resfríos, estómago, dolores } \\
\text { menstruales, afrodisíaca. }\end{array}$ \\
\hline Romero & 14 & $\begin{array}{l}\text { Rosmarinus officinalis } \mathrm{L} \text {. } \\
\text { LAMIACEAE }\end{array}$ & Parte aérea & $\begin{array}{c}\text { Dolores de cabeza, vértigo, relajante, } \\
\text { reduce la presión alta, carminativa (int.), } \\
\text { mejora el cutis, disminuye pérdida de } \\
\text { cabello (ext.). }\end{array}$ \\
\hline Rosa mosqueta & 1 & $\begin{array}{l}\text { Rosa moschata Mill. } \\
\text { ROSACEAE }\end{array}$ & Parte aérea & $\begin{array}{l}\text { Laxante, combate debilidad y fatiga, } \\
\text { diurética, antioxidante, defensa del } \\
\text { sistema inmunológico. }\end{array}$ \\
\hline Ruda & 22 & $\begin{array}{l}\text { Ruta graveolens L. } \\
\text { RUTACEAE }\end{array}$ & Parte aérea & $\begin{array}{l}\text { Dolor de estómago, dolor de oído, de } \\
\text { cabeza, dolores menstruales, mareo, } \\
\text { riñones, antidiarreico (mate), mal de ojo. }\end{array}$ \\
\hline Salvia & 4 & $\begin{array}{l}\text { Salvia officinalis L. } \\
\text { LAMIACEAE }\end{array}$ & Hojas & $\begin{array}{l}\text { Mejora digestión, ayuda circulación de } \\
\text { la sangre, diurética, sudorífera. }\end{array}$ \\
\hline Sanguinaria & 3 & $\begin{array}{l}\text { Polygonium acuminatum Kunth } \\
\text { POLYGONIACEAE }\end{array}$ & Tallos & $\begin{array}{c}\text { Alergias, acné, espinillas (ext.), } \\
\text { disminuye la presión arterial, problemas } \\
\text { de vejiga, úlceras del estómago, gota } \\
\text { (int.) }\end{array}$ \\
\hline Sen & 2 & $\begin{array}{l}\text { Cassia angustifolia } \text { Vahl } \\
\text { FABACEAE }\end{array}$ & Hojas & Laxante, adelgazante. \\
\hline Siete venas & 2 & $\begin{array}{l}\text { Plantago lanceolata } \mathrm{L} . \\
\text { PLANTAGINACEAE }\end{array}$ & Hojas & $\begin{array}{l}\text { Resfrío, asma, neumonía, colon irritable } \\
\text { (int.), cicatrizante (ext.). }\end{array}$ \\
\hline Sorona o Brea & 1 & $\begin{array}{c}\text { Tessaria absinthioides (Hook. \& } \\
\text { Arn.) DC. } \\
\text { ASTERACEAE }\end{array}$ & Hojas & Diabetes \\
\hline Té rojo & 1 & $\begin{array}{l}\text { Thea sinensis } \mathrm{L} . \\
\text { THEACEAE }\end{array}$ & Hojas & Adelgazante \\
\hline Té verde & 5 & $\begin{array}{c}\text { Camellia sinensis }(\mathrm{L} .) \text { Kuntze } \\
\text { THEACEAE }\end{array}$ & Hojas & Adelgazante \\
\hline Tilo & 1 & $\begin{array}{l}\text { Tilia europaea } \mathrm{L} . \\
\text { MALVACEAE }\end{array}$ & Hojas & Relajante, resfríos, dolores de cabeza \\
\hline
\end{tabular}




\begin{tabular}{|c|c|c|c|c|}
\hline $\begin{array}{l}\text { Nombre } \\
\text { vernáculo }\end{array}$ & Frecuencia & $\begin{array}{l}\text { Nombre científico } \\
\text { FAMILIA }\end{array}$ & Parte usada & Aplicaciones terapéuticas \\
\hline Tola & 8 & $\begin{array}{l}\text { Fabiana barriosii Phil. } \\
\text { SOLANACEAE }\end{array}$ & $\begin{array}{l}\text { Hojas y } \\
\text { tubérculos* }\end{array}$ & $\begin{array}{l}\text { Adelgazante, diurética, desinflama } \\
\text { estómago (hoja), bronquitis aguda, tos } \\
\text { persistente, antipirético, problemas de } \\
\text { vesícula biliar (tubérculo). }\end{array}$ \\
\hline Toronjil cuyano & 3 & $\begin{array}{l}\text { Marrubium vulgare } \mathrm{L} . \\
\text { LAMIACEAE }\end{array}$ & Parte aérea & $\begin{array}{l}\text { Cólicos, estomacal, digestivo, } \\
\text { adelgazante, diabetes. }\end{array}$ \\
\hline Tusilago & 1 & $\begin{array}{l}\text { Tussilago farfara } \mathrm{L} \text {. } \\
\text { ASTERACEAE }\end{array}$ & Hojas & Expectorante, tos. \\
\hline $\begin{array}{l}\text { Viza-viza o } \\
\text { visavisa }\end{array}$ & 1 & $\begin{array}{c}\text { Trixis cacalioides(Kunth) D. Don } \\
\text { ASTERACEAE }\end{array}$ & Hojas & $\begin{array}{c}\text { Cicatrizante, heridas, quebraduras de } \\
\text { huesos }\end{array}$ \\
\hline Yerba de la Plata & 1 & $\begin{array}{l}\text { Equisetum bogotense Kunth } \\
\text { EQUISETACEAE }\end{array}$ & Parte aérea & $\begin{array}{l}\text { Diarreas, hemorragias intestinales, } \\
\text { rectales y vaginales, cálculos y } \\
\text { piedrecillas al hígado, mal aliento }\end{array}$ \\
\hline Yerba de San Juan & 2 & $\begin{array}{l}\text { Hypericum perforatum } \mathrm{L} . \\
\text { HYPERICACEAE }\end{array}$ & Parte aérea & $\begin{array}{l}\text { Ansiedad, nervios, tensión premenstrual, } \\
\text { alteraciones emocionales, colon irritable }\end{array}$ \\
\hline Zarzaparrilla & 3 & $\begin{array}{c}\text { Smilax medica Schltdl. \& Cham. } \\
\text { SMILACACEAE }\end{array}$ & Hojas y palos & $\begin{array}{l}\text { Reumatismo, diurética, enfermedades } \\
\text { de la piel }\end{array}$ \\
\hline
\end{tabular}

Fuente: Madaleno y Delatorre 2012.

*Pura ancañoco en idioma Aymara

Esto nos conduce al quinto resultado, o sea, a pesar de ser más cuantioso el número de agricultores urbanos entrevistados, son menos prolíficos sus jardines, lo que suele ser habitual en América Latina (Madaleno, 2000, Madaleno y Gurovich, 2004). Esto puede provenir del hecho de ser desértico el clima de la ciudad pesquisada, a lo que se añade la pequeñez de sus jardines (Luebert, 2011, Villagrán y Castro, 2004). Pasamos a discriminar todas las especies cultivadas en los barrios de la muestra, de la capital de Tarapacá:

1) El Aloe vera y el Aloe barbadensis son las especies más frecuentes en los jardines, con veinte y nueve menciones; 2) Las tan universales mentas (Mentha x piperita y Mentha viridis) han sido registradas en quince patios; 3 ) La ruda europea (Ruta graveolens), particularmente apreciada por los aymara, se encontró en once; 4) El paico (Chenopodium ambrosioides), usado en la medicina tradicional contra el empacho, tiene siete ocurrencias, al igual que el orégano (Origanum vulgare); 6) El tan mediterráneo romero (Rosmarinus officinalis), muy utilizado contra el reuma, tiene seis registros; 7) El europeo llantén (Plantago major), un reconocido digestivo, apareció en cinco; 8) El perejil (Petroselinum crispum), adelgazante natural, tiene cuatro registros; 9) La europea manzanilla
(Matricaria chamomilla), otro digestivo y calmante natural, posee tres registros; 10) La tropical hierbaluisa (Cymbopogon citratus), dos, así como la salvia europea (Salvia officinalis), el sudamericano cedrón (Aloysia triphylla), la bella malva rosa (Pelargonium odoratissimum) y una menta conocida como poleo (Mentha pulegium), todas ellas hierbas digestivas.

Finalmente mencionamos la Plantaginaceae siete venas (Plantago lanceolada), consumida contra ataques de asma y el resfrío, que tuvo una sola ocurrencia. Igualmente una sola vez encontramos el apio (Apium graveolens) utilizado contra los cólicos de su guagua por una madre entrevistada.

Los agricultores urbanos cultivan las hierbas de uso terapéutico con fertilización orgánica $(43,8 \%)$, mayormente tierra de hoja (10 entrevistados), abono de conejo (2), guano de caballo, de cabra o de gallina (otros dos). Solamente un par de jardineros hacía compostaje de desechos orgánicos, mientras un único entrevistado agregaba cáscara de palta al suelo de su patio trasero, como solía hacerlo en la V Región de donde había migrado a Iquique, por motivos profesionales. Fueron apenas tres $(6,2 \%)$ los cultivadores de hierbas medicinales que declararon utilizar abono químico. Hay que reseñar como sexto resultado: la mitad de los entrevistados (24 jardineros) no abonaba la tierra, hecho común entre 
los que cultivan aloe, que no se riega ni fertiliza, en Iquique.

Se ha subrayado que en la medicina tradicional aymara no se usa el aloe como cicatrizante, ya que no es planta nativa, sino el molle, tanto las hojas como la ceiba, nombrada localmente "lágrima de molle", para regenerar los tejidos cutáneos. Todavía las virtudes del aloe justifican que se comercialicen cremas, compresas, gel y tónicos confeccionados por artesanos de la región. Como antiséptico, para lavarse los ojos, se utiliza en Iquique infusión de flores de caléndula europea (Calendula officinalis), que también se toma como té. La zarzaparrilla nativa de la zona sur de Chile (Smilax medica) está prescrita para enfermedades de la piel, indiscriminadamente; sin embargo, se utiliza una profusión de hierbas (romero), viscos (aloe), frutos (palta) y legumbres (pepino), para mejorar el cutis, entre las mujeres, que comparten prescripciones domésticas para resolver los problemas de resecación que sufren, en una ciudad donde no llueve casi nunca.

\section{Tratamiento de enfermedades específicas}

La diabetes se trata en la medicina ancestral aymara con especies nativas como el culén (Otholobium glandulosum), la morera (Morus tinctoria) y la flor de yareta (Azorella compacta), cuyo uso se había ya señalado en estudios anteriores, en la capital chilena, Santiago (Madaleno, 2007). Especies endémicas del norte de Chile, como la sorona (Tessaria absinthioides), son todavía más recomendadas para tratar la diabetes, por curanderos y acopiadores de hierbas de la etnia aymara. Aquí se abre un paréntesis para esclarecer que la Tessaria sólo se prescribe en Tarapacá, mientras entre los atacameños (Villagrán $e t$ al., 1998, Parra et al., 2004), la especie absinthioides, muy prolífica en el valle del río Loa, se considera maleza y toma nombre de brea.

En cuanto a la artritis, gota y afecciones reumáticas, las prescripciones utilizan también alguna diversidad de especies, desde ajenjo (Artemisia absinthium), aloe, manzanilla y cola de caballo (Equisetum giganteum), especie europea introducida por los colonos españoles y que se aclimató muy bien en las faldas de los Andes, donde crece silvestre y se incorporó a la medicina aymara para tratar el exceso de ácido úrico, con bastante suceso, como registramos en la entrevista realizada en Sibaya.

Enfermedades graves como el cáncer, en general se tratan con fármacos de la medicina convencional.
Sin embargo, otras especies europeas como el hinojo (Foeniculum vulgare) o el ciprés (Cupressus lusitanica), de origen portugués, se utilizan para tratar el cáncer en la próstata, aplicación que tiene una planta endémica de Tarapacá, lampaya. Especies nativas de los Andes, como el pingo-pingo (Ephedra andina) y el pichi (Fabiana imbricata) son consumidas para problemas en la próstata y recomendadas por acopiadores y comerciantes, porque dicen ser los hombres más reacios a visitar el médico que las mujeres.

\section{Conclusiones}

En Iquique, el $40 \%$ de las especies consumidas son nativas de América, el 35,2\% son de origen europeo y el $24,8 \%$ asiáticas. Destaca la utilización del africano aloe en la cultura del ciudadano de Iquique, la que se usa para muchas enfermedades como cáncer, úlceras y quemaduras. Las virtudes terapéuticas de esta Xanthorrhoeaceae son reconocidas por la moderna farmacología y por la medicina convencional, lo que le da el respaldo oficial. Ross (2003) atribuye al gel propiedades de analgésico, bactericida, anticancerígeno, fungicida, antiinflamatorio, antidiabético, antipirético, antiulceroso, antiviral y cicatrizante. Por norma, los jardines de Iquique son de pequeña dimensión, son irrigados y fertilizados orgánicamente, con la excepción del aloe, que no presenta cuidados especiales.

La segunda preferencia de los iquiqueños son las mentas, tanto las cultivadas con riego en los jardines como las adquiridas en las herboristerías. Esta observación es común en la capital chilena, Santiago (Madaleno, 2007), donde la Mentha x piperita, $M$. spicata y $M$. viridis eran las más cultivadas en los huertos obreros y familiares de la comuna de La Pintana, investigados en 2002 (Madaleno y Gurovich, 2004). El tercer puesto está ocupado por la ruda europea, menos por sus virtudes terapéuticas que por su uso mágico-religioso (Madaleno 2012). Nombrada "hierba de las brujas" en los países del mar Mediterráneo, ha sido adoptada por los aymara de Chile, que la consideran el mejor de los diuréticos, aprecian sus propiedades analgésicas, pero, sobre todo, le tienen mucha fe contra el mal de ojo.

Dentro de las especies nativas las más usadas son: el cedrón (17 ocurrencias), Verbenaceae nativa de Chile y consumida como digestivo. Sigue la flor de llareta (con 15 registros), planta del altiplano, cuya ingesta es reseñable entre los diabéticos entrevistados 
en Iquique. El puesto siguiente se atribuye al matico (12 entrevistas), Scrophulariaceae chilena, con gran diversidad de aplicaciones, mayormente consumida como regulador del colesterol por los ancianos. Al igual algunos diabéticos lo prefieren a la llareta. Finalizamos con la coca (10 ocurrencias), por lo general usada como regulador de la presión arterial y para combatir el estrés, que perturba las labores de aquellos que residen en medio urbano. Erythroxylum coca es una especie de la Amazonia, siendo tradicionalmente consumida por los pueblos de los Andes para aminorar los efectos del "mal de altura", o sea, para evitar el mareo debido a la baja presión atmosférica y rarefacción del oxígeno, que se siente por encima de los 3.000 metros.

A excepción del cedrón (Aloysia triphyilla) y del paico (Chenopodium ambrosioides), que se cultivan en los jardines, las demás plantas nativas de América, preferidas por los iquiqueños, solo se compran en las farmacias, herboristerías, a mercaderes en ferias, a colectores de hierbas y en los mercados de la ciudad, el Central y el Terminal Agropecuario, donde las mujeres aymara venden a diario, o dos veces a la semana, las plantas de la cordillera de los Andes, las hierbas medicinales producidas en los oasis y los preparados ancestrales para todo tipo de malestar. Las prácticas de cura tradicionales se mezclan con las prescripciones domésticas, tanto cuanto las especies nativas se combinan con las europeas, en un sincretismo de influencias que se destina a resolver las molestias que la medicina convencional no puede tratar, por cuestiones financieras, culturales, religiosas y hasta por motivos de opción por medicinas alternativas.

\section{Literatura Citada}

Acevedo, A. y Delatorre, J.

2012 Plantas medicinales altoandinas de la I Región de Tarapacá (Chile). Sistematización de la información bibliográfica mediante monografías sobre la flora nativa con propiedades medicinales. Leipzig: Editorial Académica Española.

\section{CEPAL}

2003 Anuario Estadístico de América Latina y el Caribe 2002. Santiago de Chile: CEPAL.

Dadush, U. y Shaw, W.

2011 Juggernaut. How emerging markets are reshaping globalization. Washington: Carnegie Endowment for Peace.

Escolano, S.U.; Ortiz, J.V.; Moreno, R.

2007 Globalización y Cambios Funcionales Recientes en las Ciudades del Sistema Urbano Chileno. Cuadernos Geográficos, vol. 41, p. 33-60.

Featherstone, D.; Ince, A.; Mackinnon, D.; Strauss, K. y Cumbers, A.

2012 Progressive Localism and the Construction of Political Alternatives. Transactions of the Institute of British Geographers, vol. 37, p. 177-182.

FUCOA

2010 Hierbas Medicinales. Santiago: MINAGRI.

Gómez, S.L. y Calderón, M.P.

2011 La voz de los pobres del Norte Grande de Chile; Estudio sobre la pobreza con participación de los afectados. Revista de Geografía Norte Grande, vol. 50, p. 87-104.

INE. Censo de 2012

2012 Santiago: Instituto Nacional de Estadísticas.

Luebert, F.

2011 Hacia una fitogeografía histórica del desierto de Atacama. Revista de Geografía Norte Grande, vol. 50, p. 105-133.

Madaleno, I.M.

2011a A Comparative Study of Medicinal Plant Cultivation and Uses in Six Latin American Cities. Advances in Environmental Biology, 2, vol. 5, № 2, p. 307-314.
Madaleno, I.M.

2012 Cultivando nuestras ciudades. Actas del Congreso de Geografía Urbana de Galicia. Galicia: Universidad de Santiago de Compostela y Asociación de Geógrafos Españoles. Publicado online por Cityfarmer. Acceso en 16 de Agosto de 2012. http://www.cityfarmer.info/2012/08/14/ cultivating-our-cities-a-governance-strategy-medicinalplants-and-uses-in-latin-america/\#more-32001

Madaleno, I.M.

2007 Etno-farmacología en Iberoamérica, una alternativa a la globalización de prácticas de cura. Cuadernos Geográficos, vol. $41, \mathrm{~N}^{\circ} 2$, p. 61-95.

Madaleno, I.M.

2011b Plantas da Medicina Popular de São Luís, Brasil. Boletim do Museu Paraense Emílio Goeldi. Ciências Humanas, vol. 6, No 2 , p. 273-286.

Madaleno, I.M.

2000 Urban Agriculture in Belém, Brazil. Cities, vol. $17 \mathrm{~N}^{\circ} 1$, p. 73-77.

Madaleno, I.M. \& Gurovich, A.

2004 "Urban Versus Rural” no Longer Matches Reality: endurance of an early public agro-residential development in peri-urban Santiago, Chile. Cities, $\mathrm{N}^{\circ} 21, \mathrm{~N}^{\circ} 6$, p. 513-526.

Madaleno, I.M. and Montero, M.C.

2011 El Cultivo de Plantas Medicinales, su Comercialización y Usos Fitoterapéuticos en Argentina: Estudio de Caso-La Ciudad de Río Cuarto, Córdoba, Argentina. VIII Coloquio de Desarrollo Local.

M.B.G.

2012 Missouri Botanical Garden. www.tropicos.org Acceso el 15 de febrero.

Olavarría, M.

2004 Pobreza, Crecimiento Económico y Políticas Sociales. Santiago de Chile: Editorial Universitaria. 
Olavarría, M.

2005 Acceso a la salud en Chile. Acta Bioethica, año XI, $\mathrm{N}^{\circ}$ 1, p. 47-64.

Parra, D.G.; Charcas, J.A.; Tello, E.N.

2004 Medicina Tradicional Atacameña. San Pedro de Atacama: Lom ediciones.

Ross, I.A.

2003 Medicinal Plants of the World. Chemical constituents, traditional and modern medicinal uses. New Jersey: Humana Press.

Sánchez, A. y Morales, R.

2004 Las Regiones de Chile. Santiago: Editorial Universitaria.

Santandreu, A.; Perazzoli, A.G.; Terrile, R.; Ponce, M.

2009 Urban agriculture in Montevideo and Rosario: A response to crisis or a stable component of the urban landscape? Urban Agriculture Magazine. vol. 22, p. 12-13.
Shvarts, S.

1995 The development of the Jewish Health Care System in Palestine and Israel during the Twentieth Century. En: BERGER, N. Jews and Medicine. Religion, culture, science. New York: The Jewish Publication Society, p. 257-270.

Villagrán, C. y Castro, V.

2004 Ciencia Indígena del Norte de Chile. Santiago: Editora Universitaria.

Villagrán, C.; Castro, V.; Sánchez, G.; Romo, M.; Latorre, C. e Hinojosa, L.F.

1998 La Tradición Surandina del Desierto: Etnobotánica del Área del Salar de Atacama (Provincia de El Loa, Región de Antofagasta, Chile). Estudios Atacameños, vol. 16, p. 7-106. WHO

2009 WHO Monographs on selected medicinal plants. Geneva: World Health Organization. 